Original article

\title{
Clinical presentation and prognosis of non valvular mitral regurgitation-A single institution experience
}

\author{
G.V. Senthilnathan ${ }^{\text {a,*, V.E. Dhandapani }}{ }^{\mathrm{b}}$, Balaji Ramraj ${ }^{\mathrm{c}}$, Muthunarayanan Logaraj ${ }^{\mathrm{d}}$ \\ ${ }^{a}$ Department of Cardiology, SRM Medical College Hospital and Research Centre, Chengalpattu, 603203, Chennai, Tamil nadu, India \\ ${ }^{\mathrm{b}}$ Department of Cardiology, SRM Medical College Hospital and Research Centre, Chengalpattu, 603203, Chennai, Tamil nadu, India \\ ${ }^{\mathrm{c}}$ Department of Community Medicine, SRM Medical College Hospital and Research Centre, Chengalpattu, 603203 Chennai, Tamil nadu, India \\ d Department of Community Medicine, SRM Medical College Hospital and Research Centre, Chengalpattu, 603203, Chennai, Tamil nadu, India
}

\section{A R T I C L E I N F O}

\section{Keywords:}

Acute coronary syndrome

Ischemia mitral regurgitation

Myocardial infarction

Ejection fraction

\begin{abstract}
A B S T R A C T
Background: Non valvular, ischemic regurgitation (MR) occurs secondary to myocardial infarction or acute ischemia. The presence of ischaemic MR is associated with increased morbidity and mortality. The severity of ischaemic MR is directly proportional to the severity of the LV dysfunction causing the MR. The present study was carried out to estimate the burden, clinical presentation and prognosis of non valvular MR among patients with coronary artery disease.

Methods: This cohort study was carried out among 75 adults aged over 25 years with electrocardiographic evidence of coronary artery disease. Clinical examination and periodic electrocardiography was done for evaluating the ischemic status and echocardiogram was done to assess the status of mitral regurgitation. Ejection fraction was measured by Simpsons method and regional wall motion abnormality was qualitatively evaluated.

Results: In this study, $60 \%$ of the participants had mild MR while $22.9 \%$ had moderate MR. In correlation with STEMI, inferior/posterior wall MI with right ventricular extension showed presence of MR in $100 \%$ of the cases. There was a statistically significant difference in the incidence of congestive cardiac failure among the patients with severe MR $(66.7 \%)$ and mild MR $(25 \%)$ compared to those without CCF ( $<<0.01)$.

Conclusion: Ischemic MR is characteristically dynamic and can change substantially with changes in loading conditions. This study has laid down the basis for evaluating future role of new adjunctive surgical techniques and of percutaneous interventions.
\end{abstract}

\section{Introduction}

Non valvular mitral regurgitation (MR) often occurs secondary to valvular consequences of a left ventricular (LV) disease. This secondary MR is defined as functional MR, due to LV remodeling by idiopathic cardiomyopathy or coronary artery disease, in which case, it is referred as ischaemic MR. Ischemic mitral regurgitation (MR) essentially occurs in patients with prior myocardial infarction (MI) or acute ischemia. Following an MI, there are significant damages to the papillary muscle or adjacent myocardium, resulting in ischemia and infarction, thereby triggering MR. In such patients, MR may become more severe with adverse remodeling or subsequent ischemia. Appropriate systolic coaptation of the anterior and posterior mitral leaflets depends on normal anatomy and function of the different components of the mitral valve apparatus. The underlying functional defect in Mitral regurgitation (MR) is systolic retrograde flow from the LV to the left atrium (LA) because of the lack of adequate coaptation of the leaflets and pressure gradient between the two cavities.

It is important to understand that secondary MR is a consequential disease rather than a pure valvular disease. Ischemic MR is considered as an abridgment, characterizing a clinical situation corresponding to chronic coronary artery disease with frequently a prior history of one or more myocardial infarctions which induced progressive LV global or regional pathological remodeling, usually in the absence of reversible ischemia. The deleterious effect of ischaemic MR is probably related to different factors. The frequent acute increase in regurgitant volume raises the volume overload and contributes to further LV dilatation. In addition, frequent acute increases in ventricular wall stress are

\footnotetext{
* Corresponding author.

E-mail addresses: senthilv1@srmist.edu.in (G.V. Senthilnathan), hod.cardio.ktr@srmist.edu.in (V.E. Dhandapani), balajir5@srmist.edu.in (B. Ramraj), logarajm@ srmist.edu.in (M. Logaraj).
} 
associated with rapid QRS widening and in turn, worsening in LV dyssynchrony.

The presence of ischaemic MR is associated with increased morbidity and mortality. It has been documented that early after an acute MI, the incidence of ischemic MR ranges from $17 \%$ to $55 \%$. In $3.4 \%$ of these patients, the mitral insufficiency is severe, resulting in increased mortality. In acute myocardial infarction, MR may pre-exist or more frequently, results from the acute event through regional LV dilation and loss of contraction. When compared with patients without MR, the patients with acute infarction and MR are older, more frequently female, have more frequently a history of previous myocardial infarction and a more severe coronary artery disease. Many studies have shown that ischaemic MR is an independent predictor of cardiovascular death. The increased mortality risk relates to the quantified degree of MR. There are significant differences in the survival outcomes between patients with non ischaemic MR, moderate MR (ERO area $<20 \mathrm{~mm}^{2}$ ) or severe MR (ERO area $>20 \mathrm{~mm}^{2}$ ). ${ }^{1}$ The prognosis is worse in the latter subgroup. However, the severity of ischaemic MR is directly proportional to the severity of the LV dysfunction causing the MR. With this background, the present study was carried out to estimate the burden, clinical presentation and prognosis of non valvular MR among patients with coronary artery disease.

\section{Methodology}

\subsection{Study setting and participants}

This study was carried out as a cohort study in the Department of Cardiology of our tertiary teaching institution among those diagnosed with acute coronary syndrome for a period of 22 months. Patients aged $>25$ years and with electrocardiographic evidence of coronary artery disease were included in the study. Patients with known valvular diseases and other systemic disease like anemia and thyroid disorders were excluded. A total of 75 patients were taken up for this study.

\subsection{Ethical approval and informed consent}

Approval was obtained from the Institutional Ethics Committee prior to the commencement of the study. Each participant was explained in detail about the study and informed consent was obtained prior to the data collection.

\subsection{Data collection}

A structured proforma was used to obtain demographic information and relevant history pertaining to risk factors. Clinical examination including documentation of the vitals was carried out by a single investigator. Periodic electrocardiography was done for evaluating the ACS status and echocardiogram was done to assess the status of mitral regurgitation. Ejection fraction was measured by Simpsons method and regional wall motion abnormality was qualitatively evaluated. The diastolic dysfunction was graded according to the diastolic filling pattern $\mathrm{E} / \mathrm{A}$ ratio as given in Table 1.

The Left ventricular internal dimension during systole and diastole were studied. The severity of the mitral regurgitation was also

Table 1

Diastolic dysfunction classification.

\begin{tabular}{lll}
\hline Grade & Classification & Description \\
\hline 1 & Mild dysfunction & $\begin{array}{l}\text { Impaired relaxation with normal filling } \\
\text { pressure }\end{array}$ \\
& & Pseudonormal mitral flow pattern \\
2 & Moderate dysfunction & Reversible restrictive with high filling \\
3 & Severe reversible & pressure \\
4 & dysfunction & Irreversible restrictive with high filling \\
& Severe irreversible & pressure \\
\hline
\end{tabular}

quantitated as mild, moderate and severe depending upon the regurgitant fraction. The grading of severity of mitral regurgitation by colour Doppler ${ }^{2}$ is as follows:

Grade-1 (mild): mitral regurgitation jet area/left atrium area ratio < $20 \%$

Grade-2 (moderate): mitral regurgitation jet area/left atrium area ratio $=20-40 \%$

Grade-3 (severe): mitral regurgitation jet area/left atrium area ratio $>40 \%$

Cardiac enzymes - SGOT, CPK-MB, Trop-T. CPKMB levels $>25 \mathrm{mg} / \mathrm{dl}$ was considered to be significant. The peak CKMB levels measured after $6 \mathrm{~h}$ of presentation was taken in our study and analysis done.

\section{Results}

In this study, $13.3 \%$ of the patients were in the age group of $40-50 y e a r s, 21.3 \%$ of them between 51 and 60 years and $26.7 \%$ of the between 61 and 70 years. In total of 75 patients 47(62.7\%) are males and $28(37.3 \%)$ of them are females(Table 2). The prevalence of non valvular MR in the present study was $46.7 \%$ among 35 patients. The observed difference was statistically significant $(\mathrm{p}<0.01$ ) (Table 3 ).

The present study showed an increase in the prevalence of mild and moderate MR with the increasing age. The age group of 71-80 years predominantly showed the MR incidence of around $50 \%$ in all the three grades. Between both the sexes, males showed increased incidence of MR, with 71.4\% showing mild MR compared to the females (Table 4). Hypertension is a significant risk factor for MR wherein $66.7 \%$ of those with hypertension manifested with severe MR. The observed difference was statistically significant ( $\mathrm{p}<0.01$ ). Similarly, ejection fraction was found to be a risk factor for MR, those with $\mathrm{EF}$ of $36 \%-49 \%$ showed $100 \%$ moderate MR and $76.2 \%$ mild MR. The observed difference was statistically significant ( $\mathrm{p}<0.001)$. (Table 5 ).

The pattern of presentation of MR revealed that $60 \%$ of the participants had mild MR while $22.9 \%$ had moderate MR. In correlation with STEMI, inferior/posterior wall MI with right ventricular extension showed presence of MR in $100 \%$ of the cases. (Table 6).There was a statistically significant difference in the incidence of congestive cardiac failure among the patients with severe MR (66.7\%) and mild MR (25\%) compared to those without CCF. The observed difference was statistically significant. $(\mathrm{p}<0.01)$. (Table 7$)$.

\section{Discussion}

Ischemic mitral regurgitation (MR) is a complication of coronary heart disease; it primarily occurs in patients with a prior myocardial infarction (MI) but also may be seen with acute ischemia, a setting in which the MR typically resolves after the ischemia resolves. ${ }^{3}$ In the setting of an MI, the MR is usually due to infarction with permanent damage to the papillary muscle or adjacent myocardium; in such patients, MR may become more severe with or without superimposed ischemia. ${ }^{4}$ In the present study done among 75 patients with acute coronary syndrome (ACS), ischemic MR was present among $46.7 \%$. In INDIA, various studies have reported an incidence from $14 \%$ to $64 \%$ by

Table 2

Background characteristics of the study participants.

\begin{tabular}{llll}
\hline S. No & Characteristics & Frequency $(\mathrm{n}=75)$ & Percentage (\%) \\
\hline $\mathbf{1}$ & Age (in years) & & \\
& $40-50$ & 10 & 13.3 \\
& $51-60$ & 16 & 21.3 \\
& $61-70$ & 20 & 26.7 \\
& $71-80$ & 25 & 33.3 \\
& $>80$ & 4 & 5.3 \\
$\mathbf{2}$ & Sex & & \\
& Male & 47 & 62.7 \\
& Female & 28 & 37.3 \\
\hline
\end{tabular}


Table 3

Prevalence of non-valvular mitral regurgitation among the study participants.

\begin{tabular}{llllll}
\hline $\begin{array}{l}\text { Total no of } \\
\text { patients }\end{array}$ & $\begin{array}{l}\text { Total no } \\
\text { patients } \\
\text { having MR }\end{array}$ & Percent & $\begin{array}{l}\text { Total no patients } \\
\text { having with out } \\
\text { MR }\end{array}$ & Percent & $\begin{array}{l}\text { P } \\
\text { value }\end{array}$ \\
\hline 75 & 35 & 46.7 & 40 & 53.3 & $<0.01$ \\
\hline
\end{tabular}

Table 4

Distribution of MR according to age and sex.

\begin{tabular}{|c|c|c|c|c|c|c|}
\hline \multirow{3}{*}{ Characteristics } & \multicolumn{6}{|c|}{ MR grade } \\
\hline & \multicolumn{2}{|c|}{ Mild } & \multicolumn{2}{|c|}{ Moderate } & \multicolumn{2}{|c|}{ Severe } \\
\hline & $\mathrm{N}$ & $\%$ & $\mathrm{~N}$ & $\%$ & $\mathrm{~N}$ & $\%$ \\
\hline \multicolumn{7}{|l|}{ Age (in years) } \\
\hline $40-50$ & 1 & 4.8 & 0 & 0.0 & 0 & 0.0 \\
\hline $51-60$ & 5 & 23.8 & 1 & 12.5 & 1 & 16.7 \\
\hline $61-70$ & 5 & 23.8 & 3 & 37.5 & 1 & 16.7 \\
\hline $71-80$ & 10 & 47.6 & 4 & 50.0 & 3 & 50.0 \\
\hline$>80$ & 0 & 0.0 & 0 & 0.0 & 1 & 16.7 \\
\hline \multicolumn{7}{|l|}{ Sex } \\
\hline Male & 15 & 71.4 & 5 & 62.5 & 3 & 50.0 \\
\hline Female & 6 & 28.6 & 3 & 37.5 & 3 & 50.0 \\
\hline
\end{tabular}

Table 5

Risk factors of MR.

\begin{tabular}{|c|c|c|c|c|c|c|c|c|c|}
\hline \multirow[t]{3}{*}{ Factors } & \multicolumn{6}{|c|}{ MR grade } & \multirow{2}{*}{\multicolumn{2}{|c|}{ Total }} & \multirow[t]{3}{*}{$P$ value } \\
\hline & \multicolumn{2}{|c|}{ Mild } & \multicolumn{2}{|c|}{ Moderate } & \multicolumn{2}{|c|}{ Severe } & & & \\
\hline & $\mathrm{N}$ & $\%$ & $\mathrm{~N}$ & $\%$ & $\mathrm{~N}$ & $\%$ & $\mathrm{~N}$ & $\%$ & \\
\hline \multicolumn{10}{|c|}{ Hypertension } \\
\hline Yes & 3 & 14.3 & 3 & 37.5 & 4 & 66.7 & 10 & 28.6 & $<0.01$ \\
\hline No & 18 & 85.7 & 5 & 62.5 & 2 & 33.3 & 25 & 71.4 & \\
\hline \multicolumn{10}{|c|}{ Ejection Fraction } \\
\hline $50 \%-75 \%$ & 5 & 23.8 & 0 & 0.0 & 0 & 0.0 & 5 & 14.3 & $<0.0001$ \\
\hline $36 \%-49 \%$ & 16 & 76.2 & 8 & 100 & 0 & 0.0 & 24 & 68.6 & \\
\hline$<35 \%$ & 0 & 0.0 & 0 & 0.0 & 6 & 100 & 6 & 17.1 & \\
\hline
\end{tabular}

Table 6

Pattern of presentation of MR in total population by ECHO.

\begin{tabular}{llll}
\hline Characteristics & N & Frequency & Percentage \\
\hline Severity of MR & & & \\
Mild & 35 & 21 & 60.0 \\
Moderate & 35 & 8 & 22.9 \\
Severe & 35 & 6 & 17.1 \\
Distribution according to STEMI & & & \\
Anterior wall MI & 35 & 14 & 28.5 \\
Inferior wall MI & 28 & 11 & 28.2 \\
Inferior wall MI + Posterior wall MI & 7 & 5 & 41.6 \\
Inferior wall MI + Right Ventricular extension & 4 & 4 & 100 \\
Inferior wall MI + Posterior wall MI +Right & 1 & 1 & 100 \\
$\quad$ Ventricular extension & & & \\
\hline
\end{tabular}

2D echo evaluation. In a study by Pant $S$ et al., mild ischemic MR was found in $64.5 \%$ of patients, slightly higher than the present study. ${ }^{5}$ Similar findings were noted in a study (Bosch Xet al 2002) of 300 patients with a non-ST elevation acute coronary syndrome, MR was detected at Doppler echocardiography in $42 \%$ : the MR was mild in $63 \%$, mild to moderate in $20 \%$, and moderate or severe in $17 \%$.Acute ischemic MR was seen with increased frequency in older patients in the present study, and was associated with inferior/posterior wall infarction and with congestive cardiac failure. ${ }^{6}$

The mechanism of ischemic MR in patients with functional disease is most likely by simple annular dilatation secondary to left ventricular enlargement, which causes incomplete mitral valve coaptation associated with normal leaflet motion. ${ }^{7}$ or local left ventricular remodeling with papillary muscle displacement causing apical tethering. The pthophysiology of ischemic MR is much more complex since myocardial damage and LV dysfunction are the causes that precede MR. The consequences of MR depend on the severity of regurgitation, the driving force and the acuteness of the lesion and in turn of LA compliance. An important characteristic of ischemic MR is its dynamic component. ${ }^{8}$ The degree of MR is best defined by the effective regurgitant orifice (ERO) area. ${ }^{9}$ The regurgitation area can change during systole, especially at early and late systole. ${ }^{10}$ These changes are determined by dynamic changes of transmitralpressure contributing to valve closure. ${ }^{11}$ Another aspect of the dynamic characteristics of ischaemic MR is a possible reduction in regurgitant volume related to a reverse $\mathrm{LV}$ remodeling obtained by appropriate medical treatment. ${ }^{12}$ In patients with chronic ischaemic MR, the ERO area can also change dynamically in the daily life, in response to changes in loading conditions leading to transient episodes of increased regurgitant volume. The degree of MR at rest is unrelated to exercise-induced changes in ERO area or regurgitant volume. ${ }^{13}$ In some patients, exercise-induced changes are low. In other patients with moderate or even severe MR at rest, a decrease in ERO area can be observed with exercise and usually results from contractile reserve of the LV, in particular of the postero-basal segment and/or a reduction in intra-LV dyssynchrony. ${ }^{14}$ In contrast, $\sim 30 \%$ of patients develop a severe increase in MR and in systolic pulmonary artery pressure during exercise. The degree of exercise-induced increase or decrease in MR relates to changes in LV remodeling and valvular deformation and also to changes in $\mathrm{LV}$ and papillary muscles synchronicity.

The increased mortality risk relates to the quantified degree of MR. ${ }^{3}$ The severity of ischemic MR tends to follow the severity of the LV dysfunction causing the MR. Currently there are no studies of outcome using sophisticated measures of LV function to determine whether ischaemic MR is a predictor of outcome independent of the amount of LV dysfunction present. Supporting this concept is the lack of evidence that correction of ischaemic MR prolongs life which would be expected to occur if MR were an independent risk factor for death beyond the LV dysfunction present. In $20 \%$ of patients, an improvement in the severity of ischemic MR is observed during exercise. ${ }^{15}$ These patients have a better long-term prognosis. Such a reduction is most frequently observed in patients with contractile reserve, especially of the posterior wall, resulting in a temporary reduced distortion of the mitral valve. ${ }^{13}$

\section{Conclusion}

Chronic ischemic MR is a frequent and important complication after myocardial infarction and is associated with an increased risk of heart

Table 7

Incidence of congestive cardiac failure (CCF) in patients with ischemic MR.

\begin{tabular}{|c|c|c|c|c|c|c|c|c|c|}
\hline \multirow[t]{3}{*}{$\mathrm{CCF}$} & \multicolumn{6}{|l|}{ MR grade } & \multirow{2}{*}{\multicolumn{2}{|c|}{ Total }} & \multirow[t]{3}{*}{$P$ value } \\
\hline & \multicolumn{2}{|l|}{ Mild } & \multicolumn{2}{|l|}{ Moderate } & \multicolumn{2}{|l|}{ Severe } & & & \\
\hline & No. of Pts & $\%$ & No. of Pts & $\%$ & No. of Pts & $\%$ & No. of Pts & $\%$ & \\
\hline Yes & 0 & 0.0 & 2 & 25.0 & 4 & 66.7 & 6 & 17.1 & $<0.01$ \\
\hline No & 21 & 100 & 6 & 75.0 & 2 & 33.3 & 29 & 82.9 & \\
\hline Total & 21 & 100 & 8 & 100 & 6 & 100 & 35 & 100 & \\
\hline
\end{tabular}


failure and cardiac mortality. It is the consequence of damaged pathologically remodelled LV inducing apical and posterior papillary muscle displacement and tethered leaflets. Ischemic MR is characteristically dynamic and can change substantially with changes in loading conditions. Although medical treatment is mandatory, it is important to consider other treatment options like cardiac resynchronization therapy or surgical correction. The future role of new adjunctive surgical techniques and of percutaneous interventions is not yet determined. Clinical randomized trials are mandatory to provide guidelines with improved level of evidence.

\section{Funding}

Nil.

\section{Ethical approval}

Obtained.

\section{Declaration of competing interest}

Nil.

\section{References}

1 Piérard LA, Carabello BA. Ischaemic mitral regurgitation: pathophysiology, outcomes and the conundrum of treatment. Eur Heart J. 2010;31(24):2996-3005.

2 Lancellotti P, Tribouilloy C, Hagendorff A, et al. Recommendations for the echocardiographic assessment of native valvular regurgitation: an executive summary from the European Association of Cardiovascular Imaging. Eur Heart $J$. 2013;14:611-644.

3 Grigioni F, Enriquez-Sarano M, Zehr KJ, Bailey KR, Tajik AJ. Ischemic mitral regurgitation: long-term outcome and prognostic implications with quantitative Doppler assessment. Circulation. 2001;103:1759-1764.
4 Uemura T, Otsuji Y, Nakashiki K, et al. Papillary muscle dysfunction attenuates ischemic mitral regurgitation in patients with localized basal inferior left ventricular remodeling: insights from tissue Doppler strain imaging. J Am Coll Cardiol. 2005;46: 113-119.

5 Pant S, Neupane P, Pant OB, et al. Mild functional ischemic mitral regurgitation following acute coronary syndrome: a retrospective study. Heart Views. 2011;12(3): 93-98.

6 Bosch X, Casanovas N, Miranda-Guardiola F, et al. Long-term prognosis of women with non-ST-segment elevation acute coronary syndromes. a case-control study. Rev Esp Cardiol. 2002;55(12):1235-1242.

7 Yiu S, Enriquez-Sarano M, Tribouilloy C, Seward J, Tajik A. Determination of the degree of functional mitral regurgitation in patients with systolic left ventricular dysfunction: a quantitative clinical study. Circulation. 2000;102:1400-1406.

8 Yoran C, Yellin EL, Becker RM, Gabbay S, Frater RW, Sonnenblick EH. Dynamic aspects of acute regurgitation: effects of ventricular volume, pressure and contractility on the effective regurgitant orifice area. Circulation. 1979;60:170-176.

9 Olson L, Subramanian R, Ackermann D, Orszulak T, Edwards W. Surgical pathology of the mitral valve: a study of 712 cases spanning 21 years. Mayo Clin Proc. 1987;62: $22-24$.

10 Yellin E, Yoran C, Sonnenblick E, Gabbay S, Frater R. Dynamic changes in the canine mitral regurgitant orifice area during ventricular ejection. Circ Res. 1979;45: $677-683$.

11 Schwammenthal E, Chen C, Benning F, Block F, Breithardt G, Levine R. Dynamics of mitral regurgitant flow and orifice area. Physiologic application of the proximal flow convergence method: clinical data and experimental testing. Circulation. 1994;90: 307-322.

12 Rosario LB, Stevenson LW, Solomon SD, Lee RT, Reimold SC. The mechanism ofdecrease in dynamic mitral regurgitation during heart failure treatment: importance of reduction in the regurgitant orifice size. J Am Coll Cardiol. 1998;32: 1819-1824.

13 Lancellotti P, Lebrun F, Piérard LA. Determinants of exercise-induced changes in mitral regurgitation in patients with coronary artery disease and left ventricular dysfunction. J Am Coll Cardiol. 2003;42:1921-1928.

14 Lancellotti P, Piérard LA. Chronic ischaemic mitral regurgitation: exercise testing reveals its dynamic component. Eur Heart J. 2005;26:1816-1817.

15 Lancellotti P, Gerard PL, Pierard LA. Long-term outcome of patients with heart failure and dynamic functional mitral regurgitation. Eur Heart J. 2005;26(15): $1528-1532$. 